\title{
The Toxicologist's Role in Community Outreach: Engaging Fellows in Education and Advocacy Efforts
}

\author{
G. A. Beauchamp ${ }^{1,2}$
}

Published online: 21 December 2015

(C) American College of Medical Toxicology 2015

In 1953, the first poison control center in the U.S. was formally recognized, and subsequently became a focal point for the efforts of physicians spearheading public education and prevention efforts surrounding the dangers of accidental poisonings in children [1-3]. While our poison control centers (PCC) and many of our senior colleagues continue to provide community service through advocacy and educational outreach, many fellowships focus primarily on the management of poisoning emergencies and education of healthcare providers [4]. Care of the poisoned patient is at the heart of what we do as toxicologists, but poisoning prevention advocacy and community education are truly the foundation of what we do. By identifying opportunities for advocacy and community outreach amenable to fellow participation, we can provide fellows with more experiences and a broader perspective of what it means to be a toxicologist.

As we explore ways to engage toxicology trainees in education and outreach efforts, we should examine recent and ongoing poisoning prevention advocacy efforts. An inspiring ACMT Ellenhorn Lecture "From Bedside to Advocacy," delivered at the 2015 North American Congress of Clinical Toxicology (NACCT) by Dr. Fred Henretig, outlined the journey from a single case of pediatric laundry pod exposure managed at the bedside to the resultant national promotion of public education and advocacy of poisoning prevention efforts

G. A. Beauchamp

beauchamp.gillian@gmail.com

1 Department of Emergency Medicine, Oregon Health and Science University, Campus Services Building 559, 3181 SW Sam Jackson Park Road, Portland, OR 97239, USA

2 Oregon Poison Center, Portland, OR, USA
[5]. Dr. Lewis Goldfrank's recent presentation on "The Importance of Toxicologic Research in Global Health" at NACCT was equally motivating, calling for a commitment to global health efforts and advocating for prevention by generating and sharing of knowledge while engaging in respectful partnerships with national and international organizations [6]. Fellows need to consider that improving public health lies at the root of toxicology, and participating in education and outreach locally and nationally during training is a key first step in engaging in this commitment globally.

Poison control centers have traditionally been the primary exposure fellows have to community outreach and poisoning prevention advocacy. Toxicology-trained educators at PCCs provide the public with relevant toxicology information based on toxico-surveillance of the communities they serve [7, 8]. They serve a large and diverse audience including medical personnel, individuals concerned about potential toxicological exposures, policy makers, and news media professionals. Examples of PCC interactions with this community which could be undertaken by fellows include volunteering at safety fairs, developing protocols for local emergency medical services (EMS) agencies, collaborating with local health agencies during outbreaks, and providing education on emerging exposures at training events for public service professionals, such as fire and EMS providers. Medical directors and PCC directors serve as experts in developing content for PCC websites, create educational campaigns based on epidemiological trends, participate in news media interviews, and present both direct and indirect education for the community. Many of these directors also serve on regional and state committees, where they lobby the legislature for continued PCC funding or advocate for legislation supporting poisoning prevention. Most PCCs welcome fellow collaboration and provide mentorship for interested fellows in any of these facets of community education. 
Involvement in professional organizations is another means to participating in structured education, outreach, and advocacy activities outside of the PCC realm. The ACMT Public Affairs Committee (PAC), for example, provides a link between the toxicology community and the greater public through social media, press releases, and a vibrant online presence. Subcommittees within the PAC track and share toxicology content via social media, work with members to draft and disseminate press releases, and develop content for the public outreach portions of the ACMT website. This includes development of the content for "FAQs" and "Ask-A-Toxicologist" to promote public education surrounding medication toxicity, occupational exposures, drugs of abuse, and natural toxins. ACMT's "Toxicology in the News," is an online resource that provides the public with a list of news reports from multiple sources, and important food and drug safety alerts [9]. ACMT is very encouraging of fellow involvement in committees, though fellows should not feel those are the only opportunities for exploration of outreach and poisoning prevention advocacy during fellowship. By pursuing mentorship within any professional organization (example: AACT, AAPCC, ACMT, ATSDR, CDC, etc.), fellows can gain experience with broader aspects of toxicology, develop skills for creating organizationdriven educational content, and have a broader understanding of the variety of ongoing advocacy efforts.

As fellows develop expertise in the management of poisoning emergencies, clinical management can provide a number of outlets for fellows to serve as topic experts. Hospital and PCC public relations departments provide mentored opportunities for fellows to serve as toxicology content experts. Fellows can offer their service as speakers at educational sessions geared towards members of the public. School and recreational organization events are also ideal places to provide public education. For example, a fellow treating a pediatric envenomation at the bedside could offer their services as an educator during outreach activities at that child's school or recreational facility. Further, bedside interactions with EMS personnel regarding a poisoning case could prompt the development and coordination of fellow-driven continuing education for such local providers.

Toxicologists have an obligation to members of the public, health educators, policy makers, emergency medical services personnel, and healthcare professionals, who all consume information about toxicological exposures from a variety of sources. As content experts, toxicologists are uniquely poised to develop and deliver credible outreach and educational resources. Training programs should encourage fellows to take advantage of the multitude of opportunities for community outreach, as such involvement complements the clinical and research aspects of fellowship training. Ultimately, developing skills in advocacy and community education can enhance one's career in toxicology and promote professional development through service.

Acknowledgments Special thanks to Dr. Michelle Ruha for her input during the writing of this editorial and to Dr. Christine Murphy for her assistance with this editorial.

\section{Compliance with Ethical Standards}

Conflict of Interest The author declares that she has no conflict of interest.

\section{References}

1. Arena JM. The pediatrician's role in the poison control movement and poison prevention. Am J Dis Child. 1983;137(9):870-3.

2. Burda AM, Burda NM. The nation's first poison control center: taking a stand against accidental childhood poisoning in Chicago. Vet Hum Toxicol. 1997;39(2):115-9.

3. Spiller HA, Griffith JRK. The value and evolving role of the U.S. Poison Control Center System. Public Health Rep. 2009;124(3): 359-63.

4. Schier JG, Rubin C, Schwartz MD, Thomas JD, Geller RJ, Morgan $\mathrm{BW}$, et al. Public health partnerships in medical toxicology education and practice. Am J Prev Med. 2010;38(6):667-74.

5. Henretig FM. ACMT Ellenhorn Lecture 2015: from bedside to advocacy. San Francisco, California: Lecture presented at North American Congress of Clinical Toxicology; 2015.

6. Golfrank LR. Keynote presentation: the importance of toxicologic research in global health. San Francisco, California: Lecture presented at North American Congress of Clinical Toxicology; 2015.

7. Krenzelok EP. The use of poison prevention and education strategies to enhance the awareness of the poison information center and to prevent accidental pediatric poisonings. J Toxicol Clin Toxicol. 1995;33(6):663-7.

8. American Association of Poison Control Centers. Available at: http:// www.aapcc.org/alerts/. Accessed October 13, 2015. ACMT Media Portal.

9. ACMT Media Portal. Available at: http://www.acmt.net/public media.html. Accessed October 13, 2015. 